\title{
Congenital defects and work in pregnancy
}

\author{
A D MCDONALD, J C MCDONALD, B ARMSTRONG, N M CHERRY, R CÔTÉ, \\ J LAVOIE, A D NOLIN, D ROBERT \\ From the Institut de Recherche en Santé et en Sécurité du Travail du Québec, Montreal, Québec H3A 3C2, \\ Canada
}

ABSTRACT The risk of congenital defect was examined in 47913 pregnancies of women employed for 15 hours a week or more at time of conception. The rate of defects of all types per 1000 births in this series was 25.0; 1.8 from defects classified as chromosomal (group A), 10.8 as developmental (group B), and 12.5 as musculoskeletal (group C). Some evidence of an excess in the risk ratio $(p<0.05)$ was found in the services sector and in four occupations-agriculture and horticulture (2.61), telephone and postal clerks (1.74), a miscellaneous group of service jobs (1.68), and receptionists and information clerks (1.47); excesses of lower statistical significance $(\mathrm{p}<0 \cdot 1)$ were found in those engaged in plastics and rubber manufacture $(2 \cdot 02)$ and in child minders $(1 \cdot 84)$. There were two cases of tracheo-oesophageal fistula - a rare defect - among eight defects ( 1.32 expected) in agriculture and horticulture. Overall, the distribution of risk ratios in the 60 occupations examined was not significantly heterogeneous. Analysis of chemical exposure profiles for each occupational group showed no evidence of any increased risk, perhaps due to lack of sensitivity and discrimination in this method of exposure estimation. In 152 pregnancies of doctors and nurses who had administered antineoplastic drugs in the first month eight defects, miscellaneous in type, were observed compared with 4.05 expected $(p=0.05)$. Special study of musculoskeletal defects and work demands showed some evidence of an association with a long working week ( $\geqslant 46$ hours) but no other ergonomic factor. With these few exceptions the survey failed to identify appreciable risk of congenital defect related to occupation.

In a survey of occupational factors and outcome of pregnancy 56067 women were interviewed over a two year period (1982-4) after delivery or spontaneous abortion in 11 obstetrical units in Montreal. Questions were asked about recently completed and all previous pregnancies-104 649 in all. The first in this series of reports outlined the risk of four main adverse outcomes (spontaneous abortion, stillbirth, congenital defect, and low birth weight) in six main employment sectors and 60 occupational groups. ${ }^{1}$ A $10 \%$ increase in risk of spontaneous abortion was observed in sales and services sectors and between $10 \%$ and $20 \%$ increases in risk of congenital defects and low birth weight in services and manufacturing. Overall, managerial, health, and clerical sectors showed no increased risk of any of these outcomes.

Subsequent analyses have addressed each of the main types of adverse outcome separately. In a report on fetal death at different stages of pregnancy - early, mid, and late abortion, and stillbirth-work

Accepted 14 August 1987 requirements and environmental conditions as described by the respondents and exposures to chemicals estimated from other sources were examined in the six main occupational sectors. ${ }^{2}$ Spontaneous abortion was related to lifting heavy weights, other physical effort and standing, and stillbirth to physical effort and vibration. Exposure to organic solvents, mainly in manufacturing, was associated with an increased risk of stillbirth and weakly with spontaneous abortion. Preterm birth and low birth weight were related in most sectors to heavy lifting and long hours of work and in some to changing shift work and noise. ${ }^{3}$

Congenital defects have been the subject of a further field survey in which the best possible estimates of exposure to chemicals were made for 301 matched case-referent pairs by two hygienists who visited all workplaces considered to have potential exposures to chemicals. ${ }^{4}$ Of nine chemical categories, including all grades of exposure, only aromatic solvents (especially toluene) were associated with an excess of defects (odds ratio $2 \cdot 3, p=0.04$ ), particularly of the kidney and urinary tract. An unexplained and possibly coincidental excess of hydronephrosis, ureteral obstruc- 
tion, and vesicoureteric reflux was also noted in women who had used a visual display unit in pregnancy. $^{5}$

The present report gives findings from a comprehensive analysis of congenital defects by occupational title, chemical exposure patterns, and work requirements. In particular, defects were studied in relation to occupation and exposures in early pregnancy for evidence of a teratogenic effect and to work requirements and postures in later pregnancy for evidence that they contributed to musculoskeletal abnormalities, perhaps by pressure on the uterus.

Fetal death and prematurity, especially the latter, are known to be importantly related to certain nonoccupational factors such as educational level and smoking habit, which are also associated with type of employment. Allowance had therefore been made for these and other potentially confounding variables by means of logistic regression in all previous analyses. Expected numbers of pregnancies with adverse outcomes were compared with numbers observed as ratios of observed to expected. Congenital defects differ from fetal death and prematurity in that they comprise many syndromes each with its own epidemiological pattern. For example, neural tube defects rise in frequency with descending socioeconomic status, ${ }^{6}$ trisomies with maternal age, ${ }^{7}$ cleft lip or palate varies with ethnic group ${ }^{8}$ and congenital heart disease $^{9}$ but not congenital defects generally, with cigarette smoking. ${ }^{10}$ Thus it would be necessary to take each type of defect separately in accounting for confounding variables. We did not undertake this computationally arduous and expensive task because it seemed unlikely that it would greatly affect the results. Since defects are rare, it is generally only possible to exclude chance as a cause when the relative risk is high and in these situations marginal effects of confounding do little to change interpretation. Nevertheless, the possibility of confounding is considered informally in assessing results.

\section{Methods}

SUR VEY METHODS

Our survey methods were described fully in the first paper of the series.' Briefly, interviews were conducted from 11 May 1982 to 10 May 1984 by bilingual nurses with 56067 women who had just given birth or been treated for a spontaneous abortion in 11 hospitals, in which some $90 \%$ of Montreal deliveries take place. A standard eight page questionnaire in French or English was used which included personal, social, and detailed occupational information for the 56067 recently completed (current) pregnancies and all previous pregnancies (48 582).
SUBJECTS AND EMPLOYMENT CLASSIFICATION

All pregnancies (current and previous) in which the woman had worked for at least 15 hours a week for one employer when she became pregnant were included in the present analysis. Pregnancies in which spontaneous abortion occurred before 20 weeks of gestation were excluded. Therapeutic abortions for a classifiable abnormality were included. Occupations were coded as for the Canadian census, using four digits for occupation" and two for industrial sector. ${ }^{12}$ Sixty occupations grouped in six main sectors have been used systematically in our reports. ${ }^{1-3}$ The terms used to describe the sectors (table 1) reflect the predominant types of work undertaken by women in the constituent occupational groups (table 2).

\section{ASCERTAINMENT AND CLASSIFICATION OF} CONGENITAL DEFECTS

Defects were ascertained in current pregnancies from inspection of medical records by a physician or nurse other than the one who had interviewed the woman. For previous pregnancies, the mother was asked whether any physical or mental abnormality had been noted in the child. In cases of suspected defect in current or previous pregnancies further inquiries were made from physicians or by consulting medical records. The defects were classified by a physician into some 90 specific types. Minor abnormalities such as hydrocoele, undescended testes, skin tags, and naevi were omitted. In the case-referent study of exposures to chemicals ${ }^{4}$ musculoskeletal defects and hernias were excluded. For the present analysis the coding and classification were again reviewed to improve the specificity of types of defect and to make more comparable the findings for current and previous pregnancies. Cases with multiple defects were reclassified under the defect which appeared most serious, other defects were not used in the current analyses. Known inherited defects were excluded and those that were poorly described (less than $1 \%$ ) as also were those only apparent at a later stage of developmentfor instance, mental retardation, cerebral palsy, epilepsy, and deafness, and so recorded in previous but not current pregnancies. Interauricular and interventricular septal defects were retained if accompanied by another heart defect. Minor degrees of hypospadias were excluded as these were only reported fully in current pregnancies.

The various types of defect were grouped for analysis into three categories: group $\mathrm{A}$, with chromosomal errors; group B, developmental defects probably arising in the first few weeks of gestation; and group C, musculoskeletal defects and hernias perhaps related to influences after the first trimester. Group A comprised trisomies and a few sex chromosome anomalies. Group B included several subgroups clas- 
sified by organ system: neural tube, oral clefts, cardiac, digestive, respiratory, and genitourinary. Group C defects included club foot, congenital dislocation of the hip, and other similar malformations; hernias were also included in this group, although it was less plausible that this defect might result from factors operating late in pregnancy.

\section{EXPOSURES TO CHEMICALS}

The method of estimated exposures to chemicals has been described. ${ }^{2}$ A chemical exposure "profile" was created for as many occupations as possible. All job codes were systematically reviewed and information about exposures to chemicals obtained from the following sources: (1) case-referent studies of current pregnancies 4 and previous pregnancies (unpublished observations), (2) reports of visits made by staff of community health departments in the course of occupational health surveillance, (3) estimates made for industries in the Montreal region in a study of occupational exposure and cancer, ${ }^{13}$ and (4) texts and research reports. On the basis of this information exposure to identified chemicals was graded at three levels (1) reaching an estimated maximum of less than $30 \%$ of the threshold limit value (TLV), (2) exceeding that level, and (3) probably reaching the TLV. All three levels were used in our case-referent study ${ }^{4}$ but only exposures at levels 2 and 3 in the present analysis.

A profile was established for all six digit job categories (four digits SOC ${ }^{11}$ and two digits SIC $^{12}$ ) for which, in five pregnancies evaluated, exposures to chemicals were essentially similar. Where they were dissimilar the job category was subdivided and more data obtained for each. From these profiles four types of exposures to chemicals were identified: (1) organic solvents, (2) pesticides (including bactericides, fungicides, and herbicides), (3) anaesthetic gases, and (4) other chemicals thought capable of biological activity. The information available to us was not sufficient to subdivide the first group by type of solvent, as was done in our case-referent study ${ }^{4}$ or to break down group 4. A history was obtained at the maternal interviews of administration of antineoplastic drugs in the month after the first missed menstrual period; this was limited to women whose occupation was nurse or physician and could be considered as a fifth exposure category.

\section{STA TISTICAL METHODS}

The risk of a pregnancy in a particular occupational group resulting in a defect is expressed relative to that among all working women in the study, as a ratio of defect rates. To preserve consistency of notation with our previous reports, and for convenience of further statistical analysis (see below), we have presented these rate ratios as ratios of observed numbers $(O)$ of defects in a group to numbers expected $(E)$ if the overall rate had applied to that group. In a group comprising a small proportion of the total sample this $\mathrm{O} / \mathrm{E}$ ratio approximates well to the risk of defect in the group relative to that in other working women. Exact $p$ values (one sided) for $\mathrm{O} / \mathrm{E}$ ratios were calculated on the assumption that under the null hypothesis the distribution of $\mathbf{O}$ should approximate a Poisson distribution with mean $\mathrm{E}$. Given the rareness of defects, and the small size of any of the specific groups studied relative to all working women, this approximation should be close. Tests for heterogeneity and trends of series of $\mathrm{O} / \mathrm{E}$ ratios were made by Poisson regression techniques using the GLIM computer package. ${ }^{14}$

\section{Results}

Table 1 shows the ratios of observed $(\mathrm{O})$ to expected (E) numbers of defects by group in the six main occupational sectors. Only in the services sector was

Table 1 Observed $(O)$ to expected $(E)$ ratios $(O / E)$ of congenital defects by main occupational sector

\begin{tabular}{|c|c|c|c|c|c|c|c|c|c|c|c|c|c|c|c|}
\hline \multirow[b]{3}{*}{ Sector } & \multirow{3}{*}{$\begin{array}{l}\text { No of } \\
\text { pregnancies }\end{array}$} & \multirow{2}{*}{\multicolumn{2}{|c|}{ Chromosomal (A) }} & \multicolumn{8}{|c|}{ Developmental (B) } & \multirow{2}{*}{\multicolumn{2}{|c|}{$\begin{array}{l}\text { Musculoskeletal } \\
\text { (C) }\end{array}$}} & \multirow{2}{*}{\multicolumn{2}{|c|}{$\begin{array}{l}\text { Total } \\
\text { defects }\end{array}$}} \\
\hline & & & & \multicolumn{2}{|l|}{$\begin{array}{l}\text { Neural } \\
\text { tube }\end{array}$} & \multicolumn{2}{|c|}{$\begin{array}{l}\text { Cleft lip/ } \\
\text { palate }\end{array}$} & \multicolumn{2}{|c|}{ Cardiac } & \multicolumn{2}{|l|}{ Othert } & & & & \\
\hline & & $\bar{O}$ & $O / E$ & $O$ & $O / E$ & $O$ & $O / E$ & $O$ & $O / E$ & $O$ & $O / E$ & $O$ & $O / E$ & $O$ & $O / E$ \\
\hline $\begin{array}{l}\text { Managerial } \\
\text { Health } \\
\text { Clerical } \\
\text { Sales } \\
\text { Services } \\
\text { Manufacturing }\end{array}$ & $\begin{array}{r}9231 \\
6593 \\
19419 \\
2491 \\
3974 \\
6205\end{array}$ & $\begin{array}{r}30 \\
16 \\
21 \\
5 \\
12 \\
12\end{array}$ & $\begin{array}{l}1 \cdot 20 \\
1.37 \\
0.60 \\
1 \cdot 13 \\
1.69 \\
1.06\end{array}$ & $\begin{array}{r}19 \\
6 \\
59 \\
5 \\
11 \\
19\end{array}$ & $\begin{array}{l}0 \cdot 83 \\
0 \cdot 37 \\
1 \cdot 22 \\
0 \cdot 81 \\
1 \cdot 12 \\
1 \cdot 22\end{array}$ & $\begin{array}{r}8 \\
0 \\
23 \\
1 \\
2 \\
6\end{array}$ & $\begin{array}{l}1.03 \\
1.42 \\
0.47 \\
0.59 \\
1.19\end{array}$ & $\begin{array}{r}40 \\
17 \\
74 \\
6 \\
17 \\
24\end{array}$ & $\begin{array}{l}1 \cdot 17 \\
0.71 \\
1.02 \\
0.67 \\
1 \cdot 17 \\
0.99\end{array}$ & $\begin{array}{r}29 \\
21 \\
85 \\
6 \\
18 \\
23\end{array}$ & $\begin{array}{l}0.83 \\
0.85 \\
1 \cdot 14 \\
0.64 \\
1.20 \\
0.96\end{array}$ & $\begin{array}{r}99 \\
72 \\
249 \\
28 \\
60 \\
89\end{array}$ & $\begin{array}{l}0.86 \\
0.89 \\
1.03 \\
0.91 \\
1.22 \\
1 \cdot 13\end{array}$ & $\begin{array}{r}215 \\
132 \\
511 \\
51 \\
120 \\
173\end{array}$ & $\begin{array}{l}0.93 \\
0.81 \\
1.05 \\
0.82 \\
1.21 \\
1.09\end{array}$ \\
\hline All & 47913 & 96 & & 119 & & 40 & & 178 & & 182 & & 597 & & & \\
\hline Rate per 1000 & & $1 \cdot 79$ & & $2 \cdot 48$ & & 0.83 & & $3 \cdot 71$ & & $3 \cdot 80$ & & 12 & & & \\
\hline
\end{tabular}

* $<<0.05$

†Digestive, respiratory, renal, and urinary tract malformations. 
Table 2 Ratio of observed $(O)$ to expected $(E)$ congenital defects in 60 occupational groups within the six sectors shown in table 1

\begin{tabular}{|c|c|c|c|c|c|c|c|c|c|c|}
\hline \multirow[b]{2}{*}{ Sector } & \multirow[b]{2}{*}{ Occupation (SOC and SIC) } & \multirow[b]{2}{*}{ Pregnancies } & \multicolumn{2}{|c|}{$\begin{array}{l}\text { Defects } \\
\text { A }\end{array}$} & \multicolumn{2}{|l|}{$\boldsymbol{B}$} & \multicolumn{2}{|l|}{$C$} & \multicolumn{2}{|c|}{ Total } \\
\hline & & & $O$ & $O / E$ & $O$ & $O / E$ & $\boldsymbol{O}$ & $O / E$ & $O$ & $O / E$ \\
\hline \multirow[t]{2}{*}{1} & $\begin{array}{l}\text { Managers, lawyers }(1111-49,2341-9) \\
\text { Accountants and analysts }(1171-3) \\
\text { Other administrators }(1174-9) \\
\text { Physical scientists }(2111-2119) \\
\text { Life scientists }(2131-9) \\
\text { Architects, engineers }(2141-69) \\
\text { Mathematicians, statisticians }(2181-9) \\
\text { Social scientists }(2311-39) \\
\text { Librarians, archivists (2350-2519) } \\
\text { Teachers, university, and postsecondary } \\
\text { (2711-19, 2791-3) }\end{array}$ & $\begin{array}{r}1304 \\
993 \\
997 \\
174 \\
177 \\
257 \\
414 \\
732 \\
301 \\
519\end{array}$ & $\begin{array}{l}4 \\
\frac{2}{-} \\
\frac{1}{4} \\
\frac{1}{1}\end{array}$ & $\begin{array}{l}1 \cdot 73 \\
1 \cdot 13 \\
- \\
\overline{2} \\
\frac{2 \cdot 17}{3 \cdot 05^{*}} \\
\overline{1.06}\end{array}$ & $\begin{array}{r}11 \\
9 \\
16 \\
1 \\
4 \\
4 \\
5 \\
9 \\
1 \\
5\end{array}$ & $\begin{array}{l}0.79 \\
0.85 \\
1.54 \\
0.53 \\
2.10 \\
1.44 \\
1.14 \\
1.14 \\
0.31 \\
0.89\end{array}$ & $\begin{array}{r}16 \\
6 \\
12 \\
2 \\
1 \\
2 \\
4 \\
11 \\
5 \\
3\end{array}$ & $\begin{array}{l}1.00 \\
0.49 \\
0.97 \\
0.92 \\
0.31 \\
0.62 \\
0.78 \\
1.21 \\
1.35 \\
0.55\end{array}$ & $\begin{array}{r}31 \\
17 \\
28 \\
3 \\
5 \\
7 \\
9 \\
24 \\
6 \\
9\end{array}$ & $\begin{array}{l}0.96 \\
0.69 \\
1.14 \\
0.68 \\
0.92 \\
1.09 \\
0.88 \\
1.31 \\
0.80 \\
0.75\end{array}$ \\
\hline & $\begin{array}{l}\text { Teachers, secondary }(2733) \\
\text { Teachers, primary and other }(2731,2739 \text {, } \\
2797,2799)\end{array}$ & $\begin{array}{r}823 \\
1944\end{array}$ & $\begin{array}{l}1 \\
4\end{array}$ & $\begin{array}{l}0 \cdot 66 \\
1 \cdot 13\end{array}$ & $\begin{array}{l}10 \\
15\end{array}$ & $\begin{array}{l}1 \cdot 08 \\
0 \cdot 70\end{array}$ & $\begin{array}{r}7 \\
22\end{array}$ & $\begin{array}{l}0.66 \\
0.89\end{array}$ & $\begin{array}{l}18 \\
40\end{array}$ & $\begin{array}{l}0.84 \\
0.80\end{array}$ \\
\hline \multirow[t]{4}{*}{2} & $\begin{array}{l}\text { Physicians, dentists }(3111-3119) \\
\text { Nursing supervisors }(3130) \\
\text { Nurses (3131a) } \\
\text { Nurses, psychiatric }(3131 \mathrm{~b}) \\
\text { Nurses, operating room }(3131 \mathrm{c}) \\
\text { Nursing assistants }(3134) \\
\text { Nursing aides and orderlies (3135) } \\
\text { Physiotherapists (3137) } \\
\text { Ergotherapists, dieticians, etc (3138-9, 3152- } \\
\quad 4,3136,3162-9)\end{array}$ & $\begin{array}{r}354 \\
186 \\
2351 \\
100 \\
84 \\
533 \\
423 \\
197 \\
499\end{array}$ & $\begin{array}{l}\frac{2}{3} \\
\frac{1}{2} \\
\frac{3}{2}\end{array}$ & $\begin{array}{l}3 \cdot 12 \\
\frac{-}{0.71} \\
5 \cdot 55 \\
\overline{2 \cdot 10} \\
4 \cdot 00 \\
\frac{-}{2 \cdot 25}\end{array}$ & $\begin{array}{r}1 \\
3 \\
19 \\
2 \\
2 \\
2 \\
3\end{array}$ & $\begin{array}{l}0.26 \\
1.49 \\
0.74 \\
\overline{2.15} \\
0.34 \\
\overline{0.95} \\
0.56\end{array}$ & $\begin{array}{r}6 \\
3 \\
23 \\
2 \\
1 \\
7 \\
3 \\
3 \\
6\end{array}$ & $\begin{array}{l}1.36 \\
1.30 \\
0.78 \\
1.61 \\
0.96 \\
1.05 \\
0.57 \\
1.23 \\
0.97\end{array}$ & $\begin{array}{r}9 \\
6 \\
45 \\
3 \\
3 \\
11 \\
6 \\
5 \\
11\end{array}$ & $\begin{array}{l}1.01 \\
1.29 \\
0.76 \\
1.20 \\
1.41 \\
0.82 \\
0.57 \\
1.02 \\
0.89\end{array}$ \\
\hline & $\begin{array}{l}\text { Medical laboratory technicians (3156) } \\
\text { Radiology technicians ( } 3155) \\
\text { Dental hygienists, technicians (3157, 3158, } \\
\quad 3161)\end{array}$ & $\begin{array}{l}494 \\
170 \\
284\end{array}$ & $\frac{1}{2}$ & $\frac{1 \cdot 12}{4 \cdot 00}$ & $\begin{array}{l}3 \\
1 \\
1\end{array}$ & $\begin{array}{l}0.56 \\
0.55 \\
0.33\end{array}$ & $\begin{array}{l}4 \\
1 \\
3\end{array}$ & $\begin{array}{l}0.65 \\
0.48 \\
0.85\end{array}$ & $\begin{array}{l}8 \\
2 \\
6\end{array}$ & $\begin{array}{l}0.64 \\
0.47 \\
0.85\end{array}$ \\
\hline & $\begin{array}{l}\text { Commercial artists, photographers (3311-33, } \\
3335-9)\end{array}$ & 460 & - & - & 5 & 1.03 & 6 & 1.06 & 11 & 0.97 \\
\hline & $\begin{array}{l}\text { Writers, translators }(3351-9) \\
\text { Sports women, dancers }(3360-79,3334)\end{array}$ & $\begin{array}{l}258 \\
100\end{array}$ & $\overline{-}$ & - & 1 & $0 \cdot 25$ & $\underline{4}$ & $\frac{1 \cdot 25}{-}$ & $-^{5}$ & $\begin{array}{l}0.66 \\
-\end{array}$ \\
\hline \multirow[t]{3}{*}{3} & $\begin{array}{l}\text { Secretaries }(4110-1 \text {, industrial sectors, } 1-47) \\
\text { Secretaries }(4110-1 \text {, other sectors, } 48-99) \\
\text { Typists and clerks (4113, industrial sectors, } \\
\quad 1-47)\end{array}$ & $\begin{array}{r}1113 \\
4859 \\
112\end{array}$ & $\begin{array}{l}2 \\
2 \\
1\end{array}$ & $\begin{array}{l}0.98 \\
0.23 \\
5 \cdot 00\end{array}$ & $\begin{array}{r}11 \\
60 \\
1\end{array}$ & $\begin{array}{l}0.89 \\
1 \cdot 13 \\
0 \cdot 84\end{array}$ & $\begin{array}{r}15 \\
62 \\
3\end{array}$ & $\begin{array}{l}1 \cdot 06 \\
1 \cdot 02 \\
2 \cdot 14\end{array}$ & $\begin{array}{r}28 \\
124 \\
5\end{array}$ & $\begin{array}{l}0.98 \\
1 \cdot 01 \\
1 \cdot 79\end{array}$ \\
\hline & $\begin{array}{l}\text { Typists and clerks (4113, other sectors, } 48 \text { - } \\
\text { 99) }\end{array}$ & 515 & - & - & 5 & 0.89 & 11 & $1 \cdot 70$ & 16 & $1 \cdot 23$ \\
\hline & $\begin{array}{l}\text { Book keepers }(4130,1,5,7,9) \\
\text { Cashiers }(4133) \\
\text { Office machine operators }(4140-3) \\
\text { Storekeepers, dispatchers }(4150-9) \\
\text { Record clerks (4160-9) } \\
\text { Receptionists, information clerks }(4170-1) \\
\text { Telephone, postal, reception clerks }(4172-9) \\
\text { Specialised clerks }(4190-5) \\
\text { General office clerks }(4197) \\
\text { Other clerical workers }(4199)\end{array}$ & $\begin{array}{r}4831 \\
1974 \\
1289 \\
648 \\
340 \\
780 \\
389 \\
686 \\
1192 \\
691\end{array}$ & $\begin{array}{l}7 \\
\frac{3}{-} \\
\frac{4}{-} \\
\frac{2}{1}\end{array}$ & $\begin{array}{l}0.81 \\
0.85 \\
- \\
- \\
- \\
2.86 \\
- \\
- \\
0.94 \\
0.81\end{array}$ & $\begin{array}{r}57 \\
25 \\
21 \\
8 \\
3 \\
15 \\
8 \\
5 \\
12 \\
10\end{array}$ & $\begin{array}{l}1.09 \\
1.13 \\
1.52^{*} \\
1.15 \\
0.81 \\
1.77^{*} \\
1.89 \\
0.68 \\
0.93 \\
1.34\end{array}$ & $\begin{array}{r}54 \\
30 \\
19 \\
3 \\
5 \\
12 \\
9 \\
6 \\
11 \\
9\end{array}$ & $\begin{array}{l}0.90 \\
1.22 \\
1 \cdot 10 \\
0.37 \\
1.18 \\
1.23 \\
1.85 \\
0.71 \\
0.74 \\
1.05\end{array}$ & $\begin{array}{r}118 \\
58 \\
40 \\
11 \\
8 \\
31 \\
17 \\
11 \\
25 \\
20\end{array}$ & $\begin{array}{l}0.97 \\
1.16 \\
1.20 \\
0.68 \\
0.94 \\
1.47^{*} \\
1.74^{*} \\
0.65 \\
0.87 \\
1.16\end{array}$ \\
\hline \multirow[t]{2}{*}{4} & $\begin{array}{l}\text { Saleswomen, commodities ( } 5135) \\
\text { Other sales occupations, commodities ( } 5130 \text { - } \\
\quad 3,5141-49)\end{array}$ & $\begin{array}{r}1203 \\
849\end{array}$ & $\begin{array}{l}1 \\
1\end{array}$ & $\begin{array}{l}0.46 \\
0.66\end{array}$ & $\begin{array}{r}13 \\
5\end{array}$ & $\begin{array}{l}1.00 \\
0.55\end{array}$ & $\begin{array}{r}15 \\
6\end{array}$ & $\begin{array}{l}1.00 \\
0.57\end{array}$ & $\begin{array}{l}29 \\
12\end{array}$ & $\begin{array}{l}0.97 \\
0.57\end{array}$ \\
\hline & Sales occupations, services (5170-99) & 439 & 3 & 3.85 & 一 & 一 & 7 & $1 \cdot 30$ & 10 & 0.92 \\
\hline \multirow[t]{3}{*}{5} & $\begin{array}{l}\text { Food and beverage preparation }(6121,6129) \\
\text { Food and beverage service }(6123,6125) \\
\text { Chambermaids, cleaners, janitors }(6133 \text {, } \\
\quad 6142,6191)\end{array}$ & $\begin{array}{r}423 \\
1267 \\
613\end{array}$ & $\begin{array}{l}1 \\
2 \\
1\end{array}$ & $\begin{array}{l}1.33 \\
0.88 \\
0.92\end{array}$ & $\begin{array}{l}5 \\
9 \\
7\end{array}$ & $\begin{array}{l}1 \cdot 11 \\
0.66 \\
1.07\end{array}$ & $\begin{array}{r}5 \\
23 \\
8\end{array}$ & $\begin{array}{l}0.95 \\
1.46^{*} \\
1.06\end{array}$ & $\begin{array}{l}11 \\
34 \\
16\end{array}$ & $\begin{array}{l}1.04 \\
1.08 \\
1.05\end{array}$ \\
\hline & $\begin{array}{l}\text { Hairdressers (6143) } \\
\text { Child care workers (6147) } \\
\text { Laundry and dry cleaners (6160-6169) } \\
\text { Other service occupations (remainder } 6111- \\
\quad 6199)\end{array}$ & $\begin{array}{l}714 \\
221 \\
180 \\
436\end{array}$ & $\begin{array}{l}3 \\
1 \\
1 \\
3\end{array}$ & $\begin{array}{l}2 \cdot 32 \\
2 \cdot 56 \\
3 \cdot 12 \\
3 \cdot 90^{*}\end{array}$ & $\begin{array}{l}7 \\
4 \\
3 \\
7\end{array}$ & $\begin{array}{l}0.90 \\
1.72 \\
1.50 \\
1.52\end{array}$ & $\begin{array}{l}7 \\
5 \\
2 \\
8\end{array}$ & $\begin{array}{l}0.78 \\
1.84 \\
0.88 \\
1.49\end{array}$ & $\begin{array}{r}17 \\
10 \\
6 \\
18\end{array}$ & $\begin{array}{l}0.94 \\
1.84 \\
1.31 \\
1.68 *\end{array}$ \\
\hline & Agriculture, horticulture (7113-7719) & 120 & - & - & 6 & $4 \cdot 54^{* *}$ & 2 & $1 \cdot 31$ & 8 & $2 \cdot 61^{*}$ \\
\hline
\end{tabular}


Table 2 (cont'd) Ratio of observed $(O)$ to expected $(E)$ congenital defects in 60 occupational groups within the six sectors shown in table 1

\begin{tabular}{|c|c|c|c|c|c|c|c|c|c|c|}
\hline \multirow[b]{2}{*}{ Sector } & \multirow[b]{2}{*}{ Occupation (SOC and SIC) } & \multirow[b]{2}{*}{ Pregnancies } & \multicolumn{2}{|c|}{$\begin{array}{l}\text { Defects } \\
\end{array}$} & \multicolumn{2}{|l|}{$B$} & \multirow[t]{2}{*}{$C$} & \multirow[b]{2}{*}{$O / E$} & \multicolumn{2}{|l|}{ Total } \\
\hline & & & $\bar{o}$ & $O / E$ & $\bar{O}$ & $O / E$ & & & $\bar{o}$ & $O / E$ \\
\hline \multirow[t]{2}{*}{6} & $\begin{array}{l}\text { Manufacture (8110-8599, 9310-9519) } \\
\text { Food and drink (industrial sectors 10-12) } \\
\text { Rubber and plastic (industrial sectors 15- } \\
\text { 16) }\end{array}$ & $\begin{array}{l}320 \\
139\end{array}$ & 二 & - & $\begin{array}{l}3 \\
4\end{array}$ & $\begin{array}{l}0.85 \\
2.68\end{array}$ & $\begin{array}{l}5 \\
3\end{array}$ & $\begin{array}{l}1.24 \\
1.73\end{array}$ & $\begin{array}{l}8 \\
7\end{array}$ & $\begin{array}{l}0.98 \\
2.02\end{array}$ \\
\hline & $\begin{array}{l}\text { Leather (industrial sector 17) } \\
\text { Textiles (industrial sectors 18-19) } \\
\text { Clothing (industrial sector 24) } \\
\text { Metal, electrical (industrial sectors 29-39) } \\
\text { Other occupations (8710-9199, 9530-9919, } \\
\text { and other sectors) }\end{array}$ & $\begin{array}{r}334 \\
405 \\
3646 \\
875 \\
886\end{array}$ & $\frac{-}{9}$ & $\begin{array}{l}\overline{1.35} \\
1 \cdot 52 \\
\overline{1 \cdot 26}\end{array}$ & $\begin{array}{r}3 \\
4 \\
34 \\
11 \\
13\end{array}$ & $\begin{array}{l}0.81 \\
0.89 \\
0.94 \\
1.14 \\
1.35\end{array}$ & $\begin{array}{r}9 \\
6 \\
38 \\
14 \\
14\end{array}$ & $\begin{array}{l}2 \cdot 13^{*} \\
1.17 \\
0.92 \\
1.27 \\
1.27\end{array}$ & $\begin{array}{l}12 \\
11 \\
81 \\
25 \\
29\end{array}$ & $\begin{array}{l}1.40 \\
1.06 \\
0.97 \\
1 \cdot 13 \\
1.30\end{array}$ \\
\hline
\end{tabular}

the ratio $\mathrm{O} / \mathrm{E}$ for congenital defects significantly increased $(\mathrm{O} / \mathrm{E} 1 \cdot 21, \mathrm{p}=0.02)$. Here, the ratio for chromosomal defects (group A) was 1.69 , for developmental defects (group B) was 1.12, and for musculoskeletal defects (group C) was $1 \cdot 22$, none individually significant at a $5 \%$ level. For group $\mathrm{B}$ defects the $\mathrm{O} / \mathrm{E}$ ratio was significantly raised overall in the clerical sector $(1 \cdot 14, p=0 \cdot 02)$. In table 2 observed numbers of these groups of defect and $\mathrm{O} / \mathrm{E}$ ratios are shown for the 60 occupational groups. In four groups $\mathrm{O} / \mathrm{E}$ ratios for total defects were increased $(p<0.05)$ : agriculture and horticulture (2.61), telephone and postal clerks $(1 \cdot 74)$, a miscellaneous group of service occupations (1.68), and receptionists and information clerks (1.47). In agriculture and horticulture the excess was mainly of group B; in the two clerical groups raised ratios for total defects were found in both group B and group C. There were also two occupational groups with ratios that reached a $10 \%$ significance level: child care workers $(1.84)$ and employees in plastic and rubber industries $(2 \cdot 02)$. Occupations with significantly raised $\mathrm{O} / \mathrm{E}$ ratios $(\mathrm{p}<0.05)$ for one of the three defect groups (A, B or C) but not for all defects combined were as follows: in group $A$ social scientists $(\mathrm{O} / \mathrm{E}=3.05)$, in group $B$ office machine operators $(\mathrm{O} / \mathrm{E}=1.52)$, and in group $C$ food and beverage service $(\mathrm{O} / \mathrm{E}=1 \cdot 46)$ and leatherwork $(\mathrm{O} / \mathrm{E}=2 \cdot 13)$. Overall tests for heterogeneity between $\mathrm{O} / \mathrm{E}$ ratios in the 60 occupational groups gave only suggestive evidence for heterogeneity for all defects combined $(p=0 \cdot 10)$; none for group $C(p>$ $0 \cdot 20$ ) but stronger evidence for differences in group $A$ $(p=0.05)$ and group $B$ defects $(p=0.02)$. For group $B$ defects evidence for heterogeneity depended mainly on two strikingly low $\mathrm{O} / \mathrm{E}$ ratios: for nursing aides (observed, 0; expected, 4.55) and for sales occupations, services (observed, 0 ; expected, 4.64).

Table 3 shows the $\mathrm{O} / \mathrm{E}$ ratios for exposures to chemicals above grade 1 in intensity. There was little evidence of excess in total defects, developmental (B), or musculoskeletal $(\mathrm{C})$ defects in relation to any of these exposures and that seen in the chromosomal (A) group was based on small numbers and not specific as to type of chemical. In 152 pregnancies in doctors and nurses (96 current and 56 previous) the women reported the administration of antineoplastic drugs. Eight defects occurred in this series (4.05 expected, p $=0.05$ ): there was one chromosomal abnormality and 0.27 expected (the case was Down's syndrome), two developmental defects (hypospadias and diaphragmatic hernia) and 1.51 expected, and five musculoskeletal defects (two club foot, one congenital dislocation of hip, and two hernias) and 2.27 expected.

In table 4 are shown $\mathrm{O} / \mathrm{E}$ ratios for musculoskeletal (C) defects according to certain work requirements and postures and by the period of pregnancy at which the work was done. For club foot only the $\mathrm{O} / \mathrm{E}$ ratio for other physical effort before 20 weeks was significantly raised $(1 \cdot 54, p=0 \cdot 05)$. For hernias a raised

Table 3 Ratios of observed $(O)$ to expected $(E)$ congenital defects by estimated exposure to chemical substances at level 2 (see text) in four groups

\begin{tabular}{|c|c|c|c|c|c|c|c|c|c|}
\hline & \multirow[b]{2}{*}{ Pregnancies } & \multicolumn{2}{|c|}{ Chromosomal $(A)$} & \multicolumn{2}{|c|}{ Developmental (B) } & \multicolumn{2}{|c|}{ Musculoskeletal (C) } & \multicolumn{2}{|c|}{ All defects } \\
\hline & & $O$ & $O / E$ & $\bar{O}$ & $O / E$ & $O$ & $O / E$ & $O$ & $O / E$ \\
\hline $\begin{array}{l}\text { Solvents } \\
\text { Pesticides } \\
\text { Anaesthetic gases } \\
\text { Other chemicals }\end{array}$ & $\begin{array}{r}1778 \\
428 \\
131 \\
2124\end{array}$ & $\begin{array}{l}6 \\
1 \\
0 \\
7\end{array}$ & $\begin{array}{l}1 \cdot 87 \\
1 \cdot 30 \\
1 \cdot 83\end{array}$ & $\begin{array}{r}16 \\
4 \\
2 \\
24\end{array}$ & $\begin{array}{l}0.82 \\
1.09 \\
1.37 \\
1.05\end{array}$ & $\begin{array}{r}27 \\
2 \\
1 \\
23\end{array}$ & $\begin{array}{l}1 \cdot 21 \\
0.38 \\
0.61 \\
0.84\end{array}$ & $\begin{array}{r}49 \\
7 \\
3 \\
54\end{array}$ & $\begin{array}{l}1.09 \\
0.72 \\
0.92 \\
1.00\end{array}$ \\
\hline
\end{tabular}


$\mathrm{O} / \mathrm{E}$ ratio was found for all women who worked 46 or more hours a week $(2.46, \mathrm{p}<0.01)$ or who lifted heavy weights $(1.46, p=0.05)$. Altogether there were 71 cases of congenital dislocation of the hip among the musculoskeletal defects. In pregnancies in which women were working from weeks 28 to 31 five cases of congenital dislocation were observed in women standing eight or more hours a day compared with 2.63 expected and three cases in women working 46 or more hours a week compared with 1.40 expected, but in neither was the ratio significant at a $5 \%$ level. When these cases of dislocation were divided into five groups according to the total hours worked, an upward trend in the $\mathrm{O} / \mathrm{E}$ ratios with longer hours was apparent $(0 \cdot 81$, $0 \cdot 70,1 \cdot 07,1 \cdot 58,2 \cdot 14)$. This trend with hours worked was significant $(p=0.04)$ but no such trend emerged for standing.

\section{Discussion}

There are some differences between the present findings and those previously reported. ${ }^{1}$ The number of pregnancies studied was increased by about $15 \%$ by the addition of women who worked for 15 to 30 hours a week, the classification was slightly modified with some changes in the types of defect included and excluded, and adjustments for the effect of confounding variables were omitted. Nevertheless, when 60 groups are subjected to probability testing three would be expected to give a $p$ value of $<0.05$; thus the observed excesses in both analyses could have been due to chance.

We do not think it likely that the risks in specific occupational groups presented above would be importantly changed if allowances were formally made for non-occupational confounding variables. A multivariable analysis was carried out of three non-occupational risk factors-educational level, ethnicity, and cigarette smoking. Developmental defects, neural tube defects alone, and musculoskeletal defects were considered. Smoking had no appreciable influence on risk for any type of defect. White anglophones had about half the risk of other groups for neural tube and musculoskeletal defects, but ethnicity was not related to other developmental defects. Women who had had a college education were also half as likely to have a birth with a neural tube defect, but educational level was not related to other developmental defects or to musculoskeletal defects.

Table 4 Ratios of observed $(O)$ to expected numbers ( $E$ ) of club foot, other musculoskeletal defects, and hernias by certain work requirements and postures during three stages of pregnancy

\begin{tabular}{|c|c|c|c|c|c|c|c|}
\hline & \multirow{2}{*}{$\begin{array}{l}\text { Total No of } \\
\text { pregnancies }\end{array}$} & \multicolumn{2}{|c|}{ Club foot } & \multicolumn{2}{|c|}{$\begin{array}{l}\text { Other musculoskeletal } \\
\text { defects }\end{array}$} & \multicolumn{2}{|c|}{ Hernias } \\
\hline & & $\bar{O}$ & $O / E$ & $O$ & $O / E$ & $O$ & $O / E$ \\
\hline $\begin{array}{l}\text { Lifting heavy weights: total } \\
\text { Before } 20 \text { weeks } \\
20-27 \text { weeks } \\
28-31 \text { weeks }\end{array}$ & $\begin{array}{l}6628 \\
2964 \dagger \\
1145 \dagger \\
2519 \dagger\end{array}$ & $\begin{array}{r}55 \\
28 \\
8 \\
19\end{array}$ & $\begin{array}{l}1 \cdot 15 \\
1 \cdot 31 \\
0.96 \\
1.04\end{array}$ & $\begin{array}{r}13 \\
6 \\
4 \\
3\end{array}$ & $\begin{array}{l}0.73 \\
0.75 \\
1.29 \\
0.44\end{array}$ & $\begin{array}{r}24 \\
12 \\
2 \\
10\end{array}$ & $\begin{array}{l}1 \cdot 46^{*} \\
1 \cdot 73^{*} \\
0.67 \\
1 \cdot 53\end{array}$ \\
\hline $\begin{array}{l}\text { Other physical effort: total } \\
\text { Before } 20 \text { weeks } \\
20-27 \text { weeks } \\
28-31 \text { weeks }\end{array}$ & $\begin{array}{r}3706 \\
1714 \\
611 \\
1282\end{array}$ & $\begin{array}{r}32 \\
19 \\
8 \\
5\end{array}$ & $\begin{array}{l}1 \cdot 22 \\
1 \cdot 54^{*} \\
1 \cdot 81 \\
0 \cdot 54\end{array}$ & $\begin{array}{l}7 \\
2 \\
2 \\
3\end{array}$ & $\begin{array}{l}0.72 \\
0.43 \\
1.22 \\
0.87\end{array}$ & $\begin{array}{r}13 \\
7 \\
2 \\
4\end{array}$ & $\begin{array}{l}1 \cdot 51 \\
1.80 \\
1 \cdot 34 \\
1 \cdot 24\end{array}$ \\
\hline $\begin{array}{l}\text { Long working week } 41-45 \text { hours: } \\
\text { total }\end{array}$ & 1693 & 10 & $0 \cdot 80$ & 4 & 0.88 & 4 & 0.91 \\
\hline $\begin{array}{l}\text { Before } 20 \text { weeks } \\
20-27 \text { weeks } \\
28-31 \text { weeks }\end{array}$ & $\begin{array}{l}616 \\
462 \\
846\end{array}$ & $\begin{array}{l}6 \\
3 \\
1\end{array}$ & $\begin{array}{l}1 \cdot 35 \\
1 \cdot 80 \\
0 \cdot 16\end{array}$ & $\begin{array}{l}1 \\
0 \\
3\end{array}$ & $\frac{0.60}{1 \cdot 32}$ & $\begin{array}{l}1 \\
0 \\
3\end{array}$ & $\frac{0.65}{1 \cdot 37}$ \\
\hline $\begin{array}{l}\geqslant 46 \text { hours: total } \\
\text { Before } 20 \text { weeks } \\
20-27 \text { weeks } \\
28-31 \text { weeks }\end{array}$ & $\begin{array}{r}1699^{\prime} \\
461 \\
231 \\
846\end{array}$ & $\begin{array}{r}15 \\
5 \\
1 \\
9\end{array}$ & $\begin{array}{l}1 \cdot 22 \\
1 \cdot 11 \\
0 \cdot 86 \\
1 \cdot 36\end{array}$ & $\begin{array}{l}5 \\
0 \\
0 \\
5\end{array}$ & $\begin{array}{l}\frac{1 \cdot 72}{2} \\
\frac{7}{02}\end{array}$ & $\begin{array}{r}11 \\
5 \\
1 \\
5\end{array}$ & $\begin{array}{l}2 \cdot 46^{* *} \\
3 \cdot 21^{*} \\
2 \cdot 31 \\
2 \cdot 02\end{array}$ \\
\hline $\begin{array}{l}\text { Posture: } \\
\text { Sitting only: total } \\
\text { Before } 20 \text { weeks } \\
20-27 \text { weeks } \\
28-31 \text { weeks }\end{array}$ & $\begin{array}{l}7459 \\
2202 \\
1205 \\
4142\end{array}$ & $\begin{array}{r}63 \\
16 \\
9 \\
38\end{array}$ & $\begin{array}{l}1 \cdot 17 \\
1.05 \\
1 \cdot 03 \\
1 \cdot 27\end{array}$ & $\begin{array}{r}20 \\
6 \\
4 \\
10\end{array}$ & $\begin{array}{l}0.99 \\
1.05 \\
1.23 \\
0.89\end{array}$ & $\begin{array}{r}26 \\
9 \\
9 \\
10\end{array}$ & $\begin{array}{l}1.32 \\
1.60 \\
1.60 \\
0.94\end{array}$ \\
\hline $\begin{array}{l}\text { Standing } \geqslant 8 \mathrm{~h} / \text { day: total } \\
\text { Before } 20 \text { weeks } \\
20-27 \text { weeks } \\
28-31 \text { weeks }\end{array}$ & $\begin{array}{r}5151 \\
2599 \\
818 \\
1734\end{array}$ & $\begin{array}{r}42 \\
24 \\
7 \\
11\end{array}$ & $\begin{array}{l}1 \cdot 13 \\
1 \cdot 28 \\
1 \cdot 18 \\
0 \cdot 88\end{array}$ & $\begin{array}{r}13 \\
3 \\
3 \\
7\end{array}$ & $\begin{array}{l}0.93 \\
0.43 \\
1.36 \\
1.49\end{array}$ & $\begin{array}{r}13 \\
7 \\
2 \\
4\end{array}$ & $\begin{array}{l}0.98 \\
1.07 \\
0.90 \\
0.87\end{array}$ \\
\hline
\end{tabular}

Pregnancies mutually exclusive - that is, they are classified in the last period in which the woman was employed.

${ }^{*} \mathrm{p}<0.05 ;{ }^{* *} \mathrm{p}<0.01$. 
White anglophones constitute only a small proportion $(18 \%)$ of the sample; although they were disproportionately concentrated in white collar jobs, even in these they were heavily outnumbered by white francophones, who made up most $(65 \%)$ of the sample. Thus the effect of ethnicity would be slightly to decrease expected numbers of neural tube and musculoskeletal defects in white collar occupations and very slightly decrease them in others. Accounting for educational level would have a similar but more pronounced effect on neural tube defects only (and hence very slightly on the group of all developmental defects). Thus nonoccupational risk factors related to educational level and ethnicity could explain at least some of the deficit of neural tube defects in the managerial and health sectors, and of the excess in services and manufacturing (table 1) but are unlikely to have affected other risks more than marginally.

Having regard for the possible teratogenic effect of pesticides, the raised ratio for group $B$ defects in agriculture and horticulture $(4.54, \mathrm{p}<0.01)$, despite small numbers, is worth noting. Of the six pregnancies leading to group B defects observed (expected 1.32) in 120 pregnancies, two were of tracheo-oesophageal fistula (17 per thousand) of which there were only eight cases in the entire study $(0 \cdot 17$ per 1000). The two clerical occupations with significantly raised congenital defect ratios showed an excess in both group $B$ and group $\mathrm{C}$ defects; exposure to chemicals in these occupations seems unlikely. The same is true of the raised $\mathrm{O} / \mathrm{E}$ ratio for group $\mathrm{B}$ defects $(1 \cdot 14, \mathrm{p}=0.02)$ for the whole clerical sector.

In our case-referent study ${ }^{4}$ an association was found between exposure to identified aromatic solvents and defects, mainly of the kidney and urinary tract. In the present investigation, when workplace visits were not made, aromatic and non-aromatic solvents could not be distinguished, and no excess was found in women whose work profile included exposure to organic solvents. In estimating chemical profiles in the present analysis, however, only those reaching level 2 were retained whereas in 10 of the 18 cases and four of the eight referents in the earlier study the exposure to aromatic solvents was graded at level 1. A more important factor, however, was the detailed nature of the field inquiry in the case-referent study. Whereas a reasonable degree of confidence could be put in even low level exposures for individual women obtained blind by visits to the workplace such exposures did not seem sufficient to establish profiles for an entire occupational group.

Club foot has been described as a congenital positional foot deformity, ${ }^{8}$ with perpetuation of the intrauterine position. ${ }^{15}$ The comparatively few cases with structural changes may comprise a separate entity. ${ }^{8}$ In our survey all types and grades of club foot were considered together because there was insufficient information to do otherwise. We postulated that certain ergonomic work requirements such as sitting, standing, and various types of physical effort might increase the risk of club foot and other musculoskeletal defects by requiring postures which constrained fetal movements. We found little evidence of this, whereas long hours of work were more consistently related to these defects. This suggests that factors other than posture or effort-fatigue, for example-may be important but the mechanism is obscure.

Associations between occupation and congenital defect in any one study may occur by chance; it is useful therefore to compare our findings with those of others. In an interview survey of 989 parents of children with structural congenital defects in Georgia, United States, associations between type of defect and occupation were sought. ${ }^{16}$ Increased odds ratios were observed for cleft lip or palate in nurses and for omphalocele or gastroschisis in employees in the printing industry. We found no evidence of these associations. No cleft of the lip or palate was found in nurses (2.29 expected); the only malformations in printing workers were two cases of Fallot's tetralogy. In Finland a register was used in which defects of the central nervous system, oral clefts, and musculoskeletal defects were recorded, together with a normal referent for each. ${ }^{17}$ Women working in industry or construction had more children with neural tube and musculoskeletal defects than women in administration, professions, sales, farming, transport, communication, and services; women in transport and communication had an excess of children with clefts of the lip or palate. Within the latter group telephone operators and teachers had increased odds ratios for oral clefts. In our study no cleft of the lip or palate was found in this group but only 0.33 was expected; three clefts were found in teachers compared with 3.16 expected. The $\mathrm{O} / \mathrm{E}$ ratio for neural tube defects in the manufacturing sector in our study was raised (1.22) but not at a high level of significance $(p<0 \cdot 10)$.

We conclude that this large survey in Montreal provides little convincing evidence of teratogenic risk from work in pregnancy. At the same time, two reservations should be made. Firstly, even in this study of over 100000 pregnancies, power to detect risks in small occupational groups is limited. Thus two potentially important and statistically significant excesses were based on very small numbers: a total of eight defects observed with 4.07 expected in women who had administered antineoplastic drugs and six developmental defects observed with 1.32 expected in agriculture and horticulture workers. Secondly, the crude estimates of chemical exposure we were obliged to use were probably inadequate in a situation where 
individual experience was not necessarily that of the group.

We acknowledge the invaluable cooperation of staff members in the 11 hospital maternity units (Charles Lemoyne, Jewish General, Laval, MaisonneuveRosemont, Notre-Dame, Sacré-Coeur, Ste-Justine, St-Luc, Royal Victoria, Ste-Mary's, St-Michel). We thank our nurses and clerks for their hard work and Drs C Commandeur, S Marquis, and I Tsarevsky for their many contributions.

\section{References}

1 McDonald AD, McDonald JC, Armstrong B, et al. Occupation and pregnancy outcome. Br J Ind Med 1987;44:521-6.

2 McDonald AD, McDonald JC, Armstrong B, et al. Fetal death and work in pregnancy. $\mathrm{Br} J$ Ind Med 1988;45:148-57.

3 McDonald AD, McDonald JC, Armstrong B, Cherry N, Nolin AD, Robert D. Prematurity and work in pregnancy. Br J Ind Med 1988;45:56-62.

4 McDonald JC, Lavoie J, Côté R, McDonald AD. Chemical exposures at work in early pregnancy and congenital defect: a case-referent study. Br J Ind Med 1987;44:527-33.

5 McDonald AD, McDonald JC, Armstrong B, Nolin AD, Robert $\mathrm{D}$, Cherry NM. Work with visual display units in pregnancy. $\mathrm{Br}$ $J$ Ind Med 1988;45:509-15.
6 Horowitz I, McDonald AD. Anencephaly and spina bifida in the province of Quebec. Can Med Assoc J 1969;100:1-8.

7 Stein Z, Kline J, Susser E, Shrout P, Warburton D, Susser M. Maternal age and spontaneous abortion. In: Porter IH, Hook EB, eds. Human embryonic and foetal death. New York: Academic Press, 1980:107-27.

8 Leck I. Descriptive epidemiology of common malformations (excluding central nervous system defects). Br Med Bull 1976;32:45-52.

9 Fedrick J, Alberman ED, Goldstein H. Possible teratogenic effect of cigarette smoking. Nature 1971;231:529-30.

10 Miller RW, Brendel WB, Brown AK, et al. Effects of cigarettesmoking on the fetus and child. Report of American Academy of Pediatrics Committee on Environmental Hazards. Pediatrics 1976;57:411-3.

11 Standard occupational classification. Ottawa: Statistics Canada, 1980.

12 Standard industrial classification. Ottawa: Statistics Canada, 1980.

13 Gerin M, Siemlatycki J, Kemper H, Begin D. Obtaining occupational exposure histories in epidemiologic case-control studies. J Occup Med 1985;27:420-6.

14 Baker RJ, Nelder JA. The GLIM system: release 3. Oxford: Numerical Algorithms Group, 1978.

15 Browne D. Congenital deformities of mechanical origin. Proceedings of the Royal Society of Medicine 1936;29:1409-31.

16 Erickson JD, Cochran WM, Anderson CE. Parental occupation and birth defects. A preliminary report. Contributions to Epidemiology and Biostatistics 1979;1:107-17.

17 Hemminki K, Mutanen P, Luoma K, Saloniemi I. Congenital malformations by the parental occupation in Finland. Int Arch Occup Environ Health 1980;46:93-8.

\section{Correspondence and editorials}

The British Journal of Industrial Medicine welcomes correspondence relating to any of the material appearing in the journal. Results from preliminary or small scale studies may also be published in the correspondence column if this seems appropriate. Letters should be not more than 500 words in length and contain a minimum of references. Tables and figures should be kept to an absolute minimum. Letters are accepted on the understanding that they may be subject to editorial revision and shortening.

The journal now also publishes editorials which are normally specially commissioned. The Editor welcomes suggestions regarding suitable topics; those wishing to submit an editorial, however, should do so only after discussion with the Editor. 